\title{
INITIAL RESULTS FROM THE POLAR MAGNETIC FIELDS INVESTIGATION
}

\author{
C. T. Russell*, G. Le*, X.-W. Zhou*, P. H. Reiff**, J. G. Luhmann***, \\ C. A. Cattell†, R. L. McPherron* and M. Ashour-Abdalla* \\ *Institute of Geophysics and Planetary Physics, University of California, \\ Los Angeles, CA 90095-1567, U.S.A. \\ **Rice University, Houston, TX 77251, U.S.A. \\ ***University of California, Berkeley, CA 94720 , U.S.A. \\ †University of Minnesota, Minneapolis, MN 55455, U.S.A.
}

\begin{abstract}
The POLAR magnetic field investigation was designed to provide a high temporal resolution and high sensitivity measurement of the magnetic field along the POLAR orbit from perigee at $2 R_{E}$ to apogee at $9 \mathrm{R}_{\mathrm{E}}$. The objectives of the investigation include probing the field aligned currents that couple the solar wind interaction and the tail dynamics to the auroral ionosphere. The launch of the spacecraft on February 24, 1966 was nominal and the turn on of the instruments very successful. Comparisons with Tsyganenko's 1995 model of the external current systems show that at quiet times it reproduces the field well. POLAR is clearly observing the field aligned current systems and the ring current. On one occasion when the IMF was strongly northward and the solar wind dynamic pressure stronger than usual the high altitude, high latitude magnetosphere became filled with magnetosheath type plasma. This event was probably an encounter with the polar cusp near the cusp-magnetosheath interface.
\end{abstract}

O1997 COSPAR. Published by Elsevier Science Ltd.

\section{INTRODUCTION}

The POLAR spacecraft [Acuna et al., 1995] has finished its initial commissioning phase and is now taking regular observations with most of its instrumentation. The spacecraft was launched on February 24 with its orbital plane in the local time sector just premidnight inbound and just past noon outbound. The orbital plane crossed the noon-midnight meridian on April 10. The satellite is spin stabilized with its spin axis perpendicular to the orbit plane. Since the orbit has a high inclination, the orbital plane is nearly in the magnetic meridian. Thus, the magnetic field lies principally in the spin plane on a typical orbit in which the spacecraft stays well within the magnetosphere. Every six months the spin axis is rotated by $180^{\circ}$ for thermal reasons.

The magnetometer is performing nominally, recording data completely around the orbit from perigee at $2 \mathrm{R}_{\mathrm{E}}$ to apogee at $9 \mathrm{R}_{\mathrm{E}}$. Two projections of a typical orbit on March 28, 1996 are shown in solar magnetic coordinates in Figures $1 \mathrm{a}$ and $1 \mathrm{~b}$. Figure 1c shows the distance of the spacecraft from the Earthsun line plotted versus the distance along the Earth-sun line for the orbit on March 16, 1996. The thick trace shows the expected magnetopause position. The dashed line shows the expected position of the 


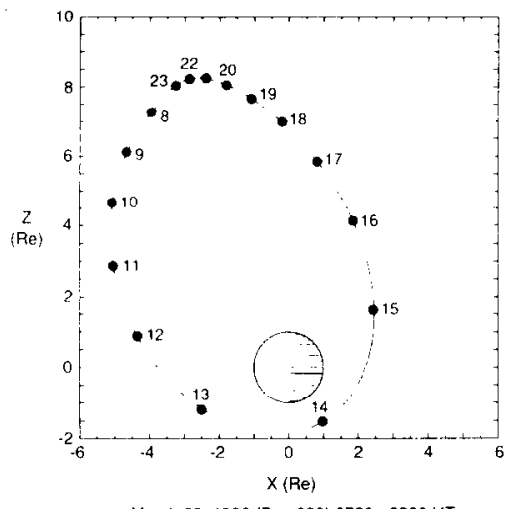

March 28, 1996 (Day 088) 0730 - 2330 UT

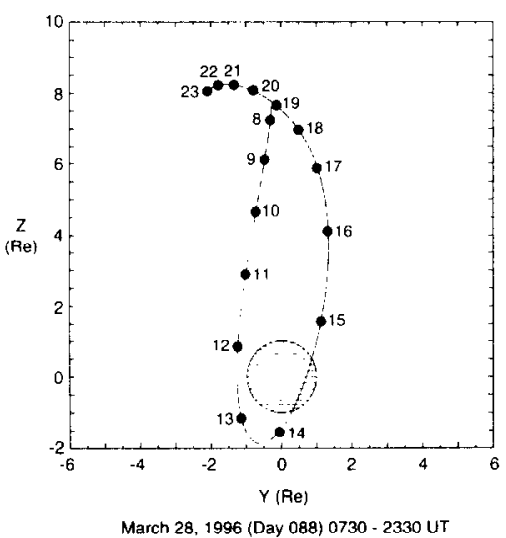

March 28, 1996 (Day 088) 0730 - 2330 UT
Fig. 1a. Noon-midnight (X-Z) projection of POLAR orbit on March 28, 1996 in solar magnetic coordinates.

Fig. 1b. Dawn-dusk (Y-Z) projection of POLAR orbit on March 28, 1996 in solar magnetic coordinates.

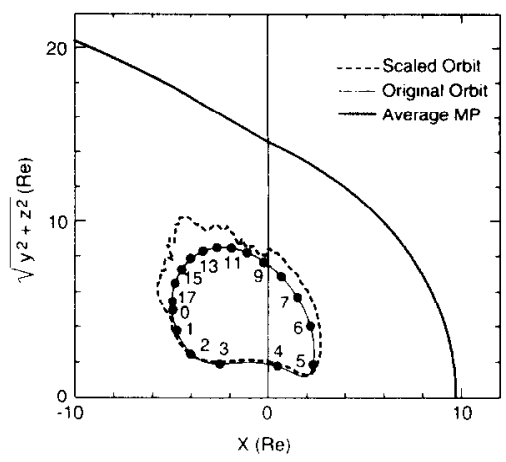

March 16, 1996 (Day 076) $0000-1730$ UT

Fig. 1c. Distance from the Earth-sun line versus distance along Earth-sun line in solar magnetospheric (or solar ecliptic) coordinates for the POLAR orbit of March 16, 1996. Nominal location of the magnetopause is shown. Scaled orbit (dashed line) shows the expected location of the spacecraft relative to the magnetopause for the solar wind dynamic pressure observed by the WIND spacecraft, obtained by scaling by the sixth root of the dynamic pressure.

spacecraft relative to the magnetopause for the solar wind pressure conditions measured by the WIND spacecraft. The orbit here has been scaled by the sixth root of the solar wind dynamic pressure. We have not accounted for the time delay between the WIND measurements and the magnetospheric arrival of the plasma and field. Here that delay was nominally $25 \mathrm{~min}$.

In this paper we examine the initial operations of the magnetic fields experiment (MFE) on the POLAR spacecraft and show examples of some of the initial results.

\section{MAGNETOMETER DESCRIPTION}

The magnetometer [Russell et al., 1995] consists of two orthogonal sensors mounted on a boom at distances of 5.225 and $7.177 \mathrm{~m}$ from the center of the spacecraft, together with electronics on the main body of the spacecraft. The inboard magnetometer has a maximum range of $46,700 \mathrm{nT}$ with a lower gain setting of $5860 \mathrm{nT}$. The outboard magnetometer has a maximum range $5525 \mathrm{nT}$ with a lower gain setting of $694 \mathrm{nT}$. The data are digitized to 16 bits. The finest digitization is $\pm 11 \mathrm{pT}$ and the coarsest is \pm 700 pT. The magnetic field is sampled at $1000 \mathrm{~Hz}$ internally and averaged to $100 \mathrm{~Hz}$. These data are distributed internally to the other instruments and made available for internal storage in the snapshot mode. 
Initially data were transmitted to Earth at a constant rate of $8.3 \mathrm{~Hz}$ or $120 \mathrm{msec}$ per sample. Every 6-s spin a sample is taken from the regular data stream just after the sun sensor crosses the solar direction. Thus, this value is within $120 \mathrm{msec}$ of the sun crossing. Later we plan to retrieve this sample from the $10 \mathrm{msec}$ data stream. In either case knowledge of the exact time of the magnetic sampling and the exact time of the sun crossing allows the reconstruction (by a small rotation) of the field vector at the time of the sun crossing (assuming no temporal variations).

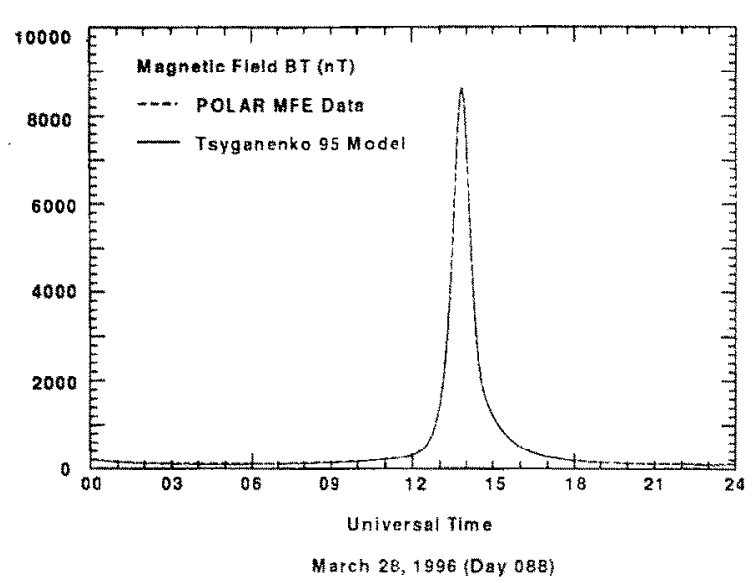

Fig. 2a. Magnetic field strength observed by the POLAR spacecraft on March 28, 1996 together with the predicted field strength obtained from the 1995 model by $N$. Tsyganenko of the external currents systems added to the IGRF 1995 internal field for epoch 1996.

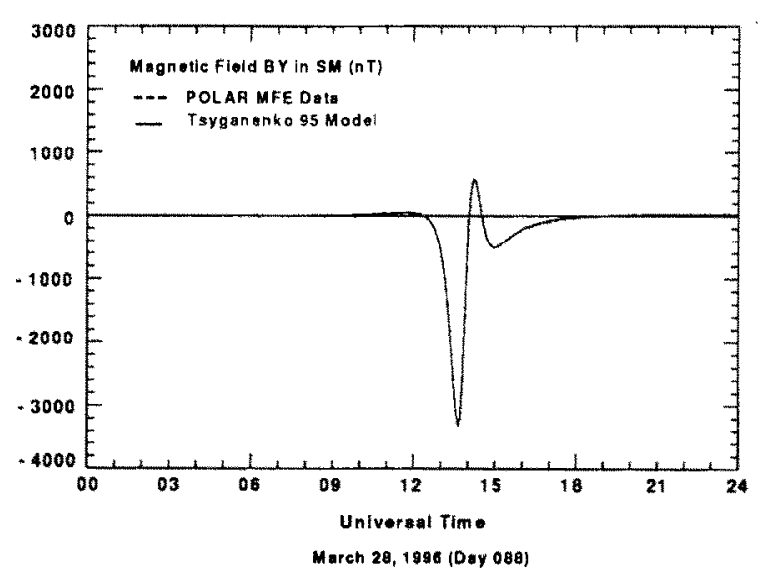

Fig. 2c. The $Y$ component of the magnetic field in SM coordinates, observed and modeled, for March 28, 1996.

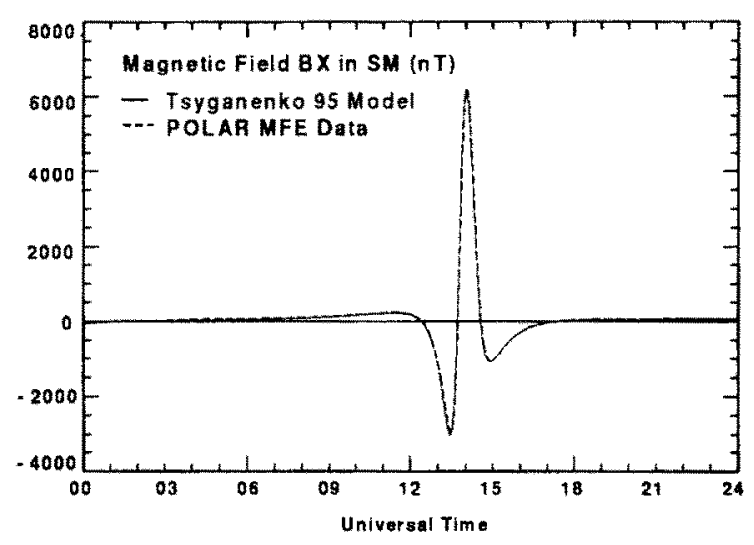

March 28, 1996 (day 088)

Fig. $2 b$. The $X$ component of the magnetic field in solar magnetic coordinates observed and modeled for March 28, 1996. In this coordinate system the $\mathrm{z}$-direction is along the northward pointing magnetic dipole axis; $y$ is perpendicular to the dipole axis and the direction to the sun; and the $x$-direction is in the plane containing the dipole axis and the solar direction completing a right handed set.

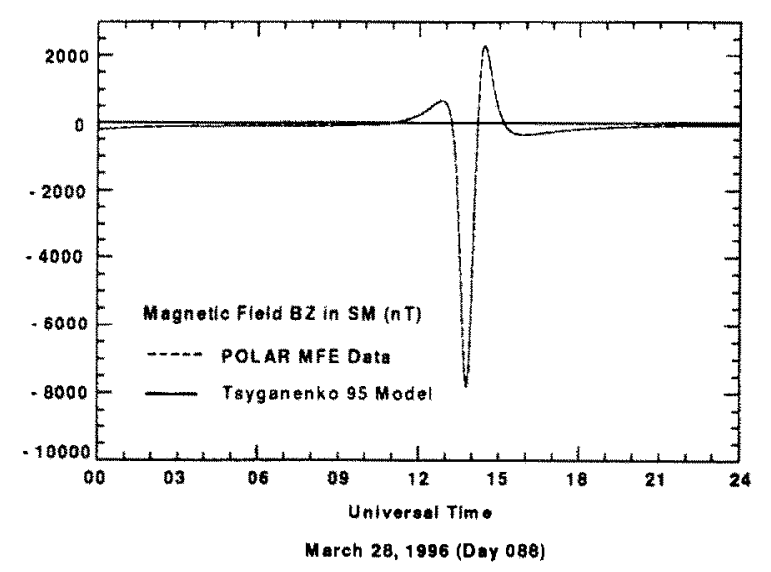

Fig. 2d. The $\mathrm{Z}$ component of the magnetic field in SM coordinates, observed and modeled, for March 28, 1996. 


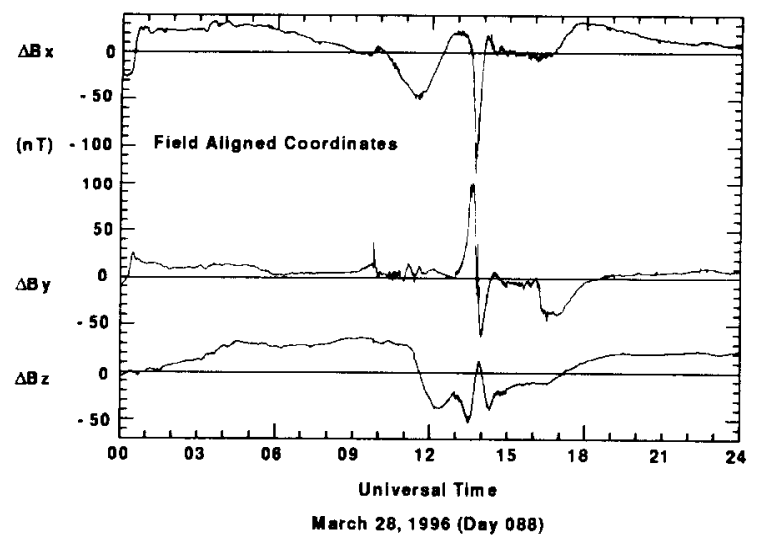

Fig. 3a. The difference between the observed magnetic field and the IGRF 1995 model expressed in field-aligned coordinates on March 28,1996 . In this system the $\mathrm{z}$ axis is along the model magnetic field; the $y$ axis is perpendicular to the field and eastward; and $x$ completes the right handed system and is roughly outward.

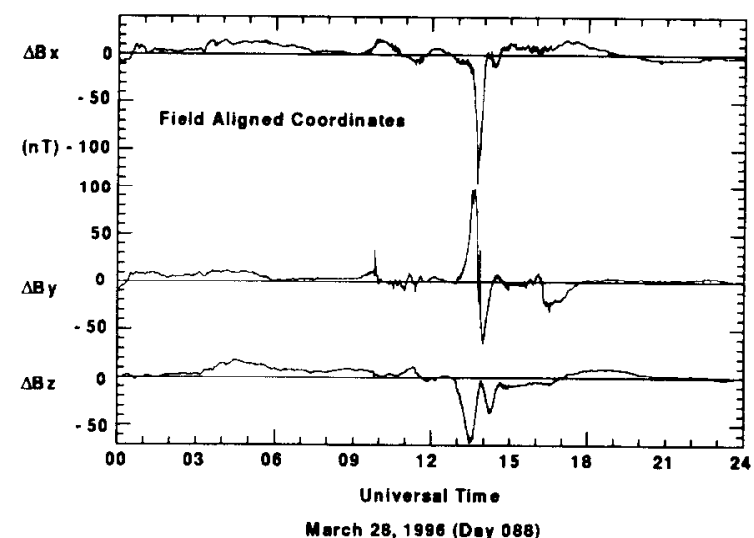

Fig. 3b. The difference between the observed magnetic field and the IGRF 1995 plus Tsyganenko 1995 models expressed in fieldaligned coordinates on March 28, 1996.

The magnetometer's internal clock runs asynchronously with the spacecraft clock. This choice was made to minimize noise sources within the magnetometer and an extremely precise and low noise instrument was thus produced. In order to synchronize the clocks a posteriori, we find specific patterns of the number of words per major frame. These occur about once every 15 minutes, and at these times, the relative timing of the magnetometer clock and the spacecraft clock are known to 1 msec. The relative clock rates change slowly with time due to aging, temperature changes and updating of the spacecraft clock. This timing information will be made available to all investigators and the clock rate will be included in the key parameter data. Information on the operation of the instrument is being maintained on the MFE world wide web page http://www-ssc.igpp.ucla.edu/polar/Welcome.html

\section{A TYPICAL ORBIT}

Figures $2 \mathrm{a}, \mathrm{b}$, c, and d show the magnetic field measured by the POLAR magnetometer in solar magnetic coordinates on March 28, 1996 together with the recent Tsyganenko 1995 magnetic field model developed and added to the International Geomagnetic Reference Field (IGRF 1995) model at epoch of date [N. A. Tsyganenko, personal communication, 1995]. Solar magnetic coordinates have their $Z$ direction along the Earth's magnetic dipole axis and the solar direction lies in the $\mathrm{X}-\mathrm{Z}$ plane. The measurements and the model are indistinguishable on this scale.

Figure $3 \mathrm{a}$ and $\mathrm{b}$ show the difference between the model and the observations rotated into a field-aligned coordinate system in which the Z-direction is along the model magnetic field, the Y-direction is eastward in the direction of the Earth's rotation and X-direction forms a right-handed set, pointing to higher Lvalues. In Figure $3 a$ only the IGRF model field has been subtracted while in Figure $3 b$ the Tsyganenko [1995] model of the external currents for $\mathrm{Kp}=3$ and $\mathrm{Dst}=0$ has been additionally removed. The residual fields are significantly better at high altitudes when the Tsyganenko model is used. At low altitudes around perigee near $1400 \mathrm{UT}$, we see a significant twist in the field in the X-Y plane perpendicular to the main field. This could be caused by a $0.8^{\circ}$ error in the pointing angle of the magnetometer or a $12 \mathrm{msec}$ 
error in the timing and we are not prepared to comment on it at present. Along the magnetic field direction there is a double depression in the field below the value expected from the model. We do not know any crrors that could lead to such a signature. Moreover, the high altitude portion of this signature is removed when the Tsyganenko model is used. This observation suggests that the feature is associated with the quiet day ring current and that the Tsyganenko model does not completely remove the effects of the ring current in the innermost magnetosphere.

\section{FIELD ALIGNED CURRENTS}

Figures $3 \mathrm{a}$ and $\mathrm{b}$ show positive and negative deviations of the $\mathrm{Y}$ or eastward component of the magnetic field at about 10 UT and 16 UT respectively. The former event is shown more clearly in Figures $4 a$ and $\mathrm{b}$ that show just the $\mathrm{Y}$ component of the magnetic field and the inclination and declination of the magnetic field respectively. The inclination angle is the angle between the field and the radius vector less $90^{\circ}$ i.e. $\mathrm{I}=\cos ^{-1}(\mathrm{Rxbx}+\mathrm{Rzbz})-90^{\circ}$ where $(\mathrm{Rx}, 0, \mathrm{Rz})$ and $(\mathrm{bx}, \mathrm{by}, \mathrm{bz})$ are unit vectors in the direction of the radius vector and the magnetic field respectively in the local dipole meridian coordinate system. The inclination may also be described as the angle between the local horizontal plane and the magnetic field direction. The declination angle is measured in the horizontal plane about the radius vector i.e. $D=\tan ^{-1}$ [by/(Rxbz - Rzbx)]. The current system responsible for the field change seen here at 0950 UT is flowing on the nightside of the Earth and appears to mark the boundary between the closed dipolar magnetic field lines of the magnetosphere proper and the expanding polar cusp field lines with perhaps only one foot on the Earth.

The second field aligned current signature shown is a similar pair of Figures, 5a and 5b showing the $y$ component of the difference field in field-aligned coordinates as well as the declination and inclination of the field. This current is flowing above the dayside hemisphere and is near the region of the expected polar cusp. The magnetic field after 1615 UT is twisted to lower declination angles as if it were being pulled more tailward at higher altitudes.

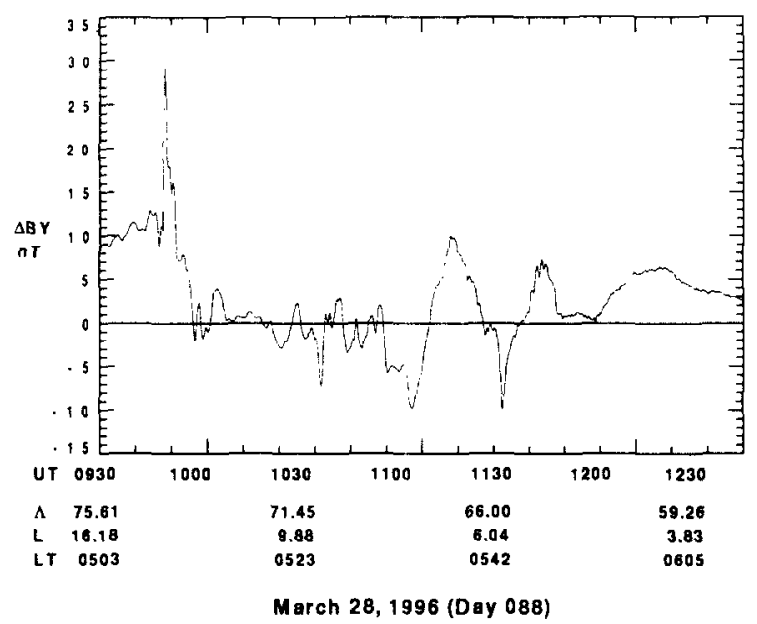

Fig. 4a. The eastward field magnetic component of the difference between the observations and the IGRF plus Tsyganenko model for the period 0930-1230 UT on March 28, 1996.

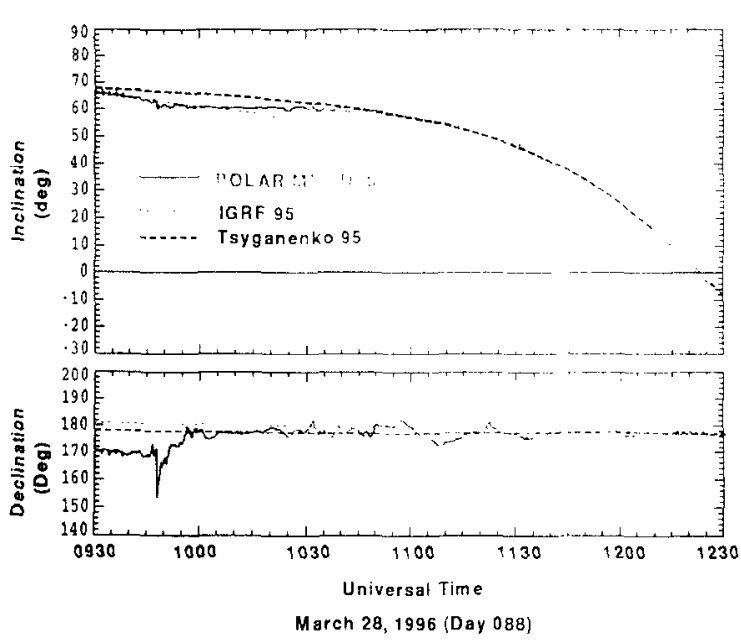

Fig. 4b. The inclination and declination of the magnetic field for the period 0930 to 1230 UT on March 28, 1996. 


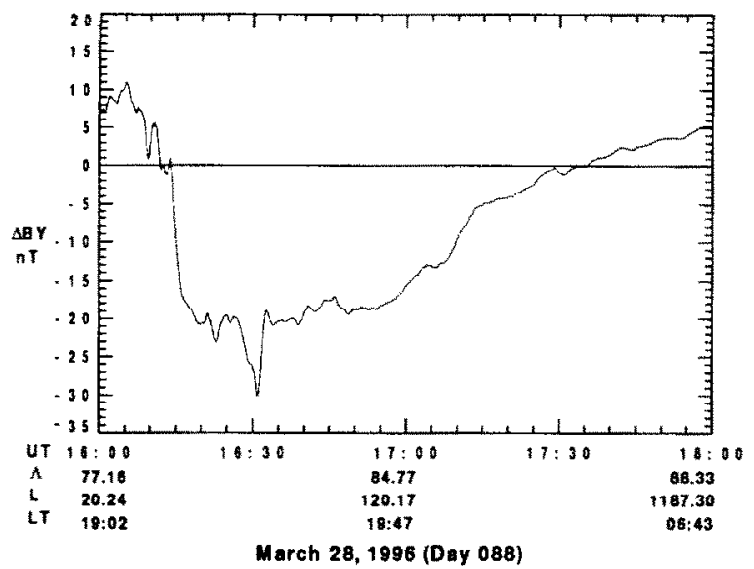

Fig. 5a. The eastward magnetic field component of the difference between the observed field and the Tsyganenko and IGRF model fields for the period 1600 to 1800 UT on March 28, 1996.

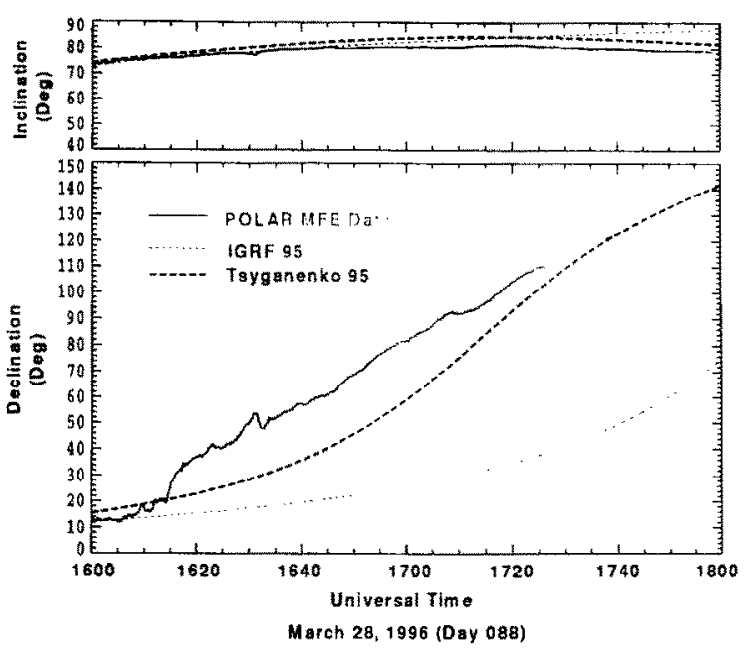

Fig. 5b. The inclination and declination of the magnetic field for the period 1600 to $1800 \mathrm{UT}$ on March 28, 1996.

\section{STRONGLY NORTHWARD IMF AND HIGH SOLAR DYNAMIC PRESSURE}

Figures $6 \mathrm{a}$ and $\mathrm{b}$ show the magnetic field in GSM coordinates and the inclination and declination angles on May 29, 1996 when the solar wind dynamic pressure was about $7 \mathrm{nPa}$ and the IMF about $12 \mathrm{nT}$ along the GSM B $\mathrm{B}_{z}$ direction (K. W. Oglivie and R. P. Lepping, personal communication, 1996). The POLAR spacecraft moved from a ZSM distance of 5 to $8 \mathrm{Re}$ moving from about $1.5 \mathrm{Re}$ to the dawn side of XZ plane to $1 \mathrm{Re}$ on the afternoon side over the period displayed here. Since the observed solar wind dynamic pressure represents an enhancement of only a factor of 3.5 and an expected compression of the magnetosphere of only $23 \%$ (to $81 \%$ of its usual radius) we would normally not expect to see the

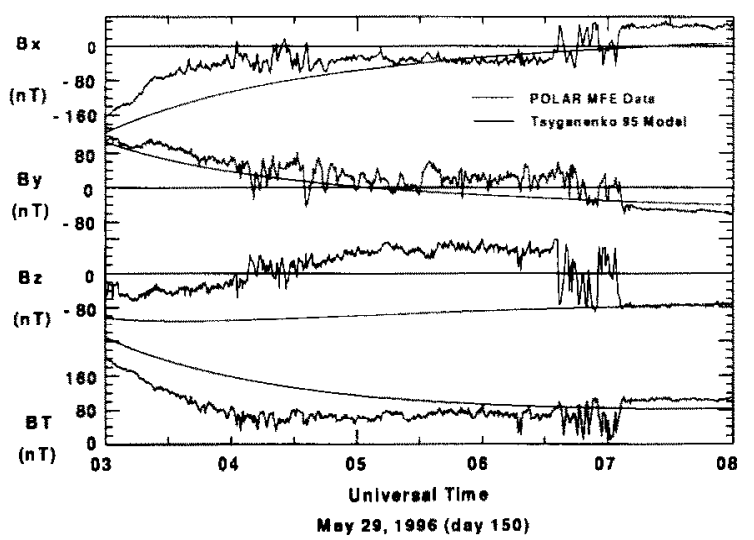

Fig. 6a. The observed magnetic field in GSM coordinates in the vicinity of the cusp on May 29,1996 . The model field is the sum of the IGRF 1995 plus Tsyganenko 1995 models.

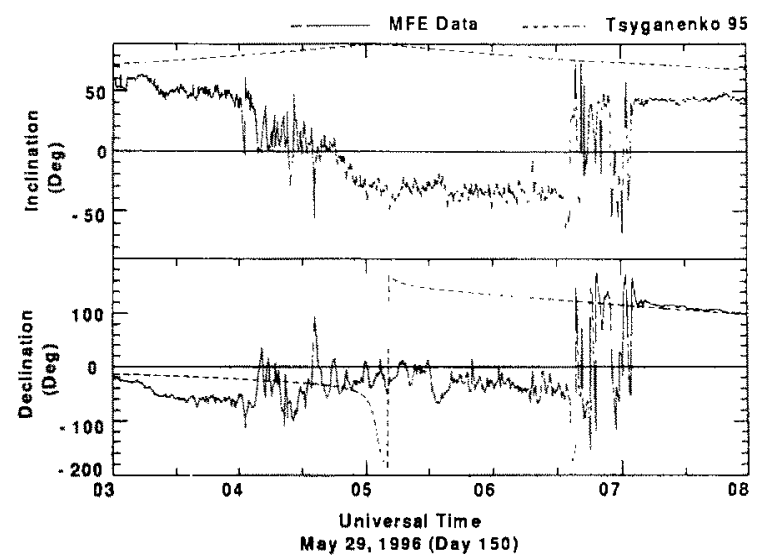

Fig. 6b. The inclination and declination of the magnetic field in the vicinity of the polar cusp on May 29, 1996. 
magnetosheath flow at the POLAR spacecraft [Zhou and Russell, 1996]. However, the magnetic field seen by POLAR was strongly depressed and twisted from its usual direction at this time. Only after 0710 UT as the spacecraft approached the noon-meridian did the field return to nearly its expected direction. At this time the declination was as expected but the inclination indicated a much more flattened polar magnetosphere. Before this time, the inclination was in fact reversed from that expected as if the magnetosheath magnetic field were draped over the magnetosphere and the spacecraft had entered this region. However, throughout this period there was no abrupt change in the field direction, indicative of a magnetopause crossing. Thus we tentatively associate this with the reconnection of magnetosheath flux to the opposite polar region. (Dungey, 1963; Russell, 1973). This hypothesis has yet to be tested with measurements from the other instruments but the shear magnitude of the depressed magnetic field and the duration of this depression indicates that the polar cusp/entry layer is very intense on this day.

\section{SUMMARY AND CONCLUSIONS}

The launch of the POLAR spacecraft has been nominal and the turn on of the instruments very successful. The magnetometer is detecting with very fine resolution field aligned current systems over both the day and night hemispheres. Comparisons with Tsyganenko's 1995 model of the external current systems show that at quiet times it reproduces the observed field at the POLAR orbit quite well. Field aligned currents produce sharp changes from the average pattern that the Tsyganenko model describes so well. The POLAR magnetometer also detects the effect of the ring current. The Tsyganenko model appears to describe these effects well at high altitudes under the quiet time conditions seen during the POLAR mission, thus far, but significant residuals appear at lower altitudes. When the IMF is strongly northward and the solar wind dynamic pressure is high, the magnetic field is strongly distorted and depressed. A possible explanation for this is high latitude reconnection perhaps in the opposite hemisphere. Whatever the explanation, the polar cusp/entry layer is filled with plasma at this time as evidenced by the strongly depressed field magnitude.

\section{ACKNOWLEDGMENTS}

The authors wish to thank the engineers responsible for building the POLAR magnetometer, R. C. Snare, J. D. Means, D. Dearborn, D. Pierce, G. Barr, M. Larson and W. Greer, as well as all the individuals responsible for the success of the spacecraft but especially Ron Harten of Lockheed-Martin. This research was supported by the National Aeronautics and Space Administration under research grant NAG5-3171.

\section{REFERENCES}

Acuna, M. H., K. W. Ogilvie, D. N. Baker, S. A. Curtis, D. H. Fairfield and W. H. Mish, The Global Geospace Science program and its investigations, Space Sci. Rev., 71, 5-21 (1995).

Dungey, J. W. The structure of the exosphere, or adventures in velocity space, in Geophysics of the Earth's Environment edited by C. DeWitt, J. Hieblot, and A. Lebeau, pp505-550, Gordon Breach, New York (1963).

Russell, C. T., The configuration of the magnetosphere, in Critical Problems of Magnetospheric Physics, The Proceedings of the Joint COSPAR/AAGA/URSI Symposium Madrid, May 11-13, 1972, edited by E. R. Dyer, 1-16, IUCSTP Secretariat, Washington D.C. (1972).

Russell, C. T., R. C. Snare, J. D. Means, D. Pierce, D. Dearborn, M. Larson, G. Barr and G. Le, The GGS/POLAR magnetic fields investigation, Space Sci. Rev., 71, 563-582 (1995).

Zhou, X-W and C. T. Russell, The location of the high latitude polar cusp and the shape of the surrounding magnetopause, J. Geophys. Res. in press (1996). 